\title{
Fast trajectory correction for nonholonomic mobile robots using affine transformations
}

\author{
Quang-Cuong Pham \\ Nakamura-Takano Laboratory \\ Department of Mechano-Informatics \\ Graduate School of Information Science and Technology \\ University of Tokyo, Japan \\ cuong.phamenormalesup.org
}

\begin{abstract}
Planning trajectories for nonholonomic systems is difficult and computationally expensive. When facing unexpected events, it may therefore be preferable to deform in some way the initially planned trajectory rather than to re-plan entirely a new one. We suggest here a method based on affine transformations to make such deformations. This method is exact and fast: the deformations and the resulting trajectories can be computed algebraically, in one step, and without any trajectory re-integration. To demonstrate the possibilities offered by this new method, we use it to derive position correction, orientation correction and obstacle avoidance algorithms for three classical nonholonomic systems: the unicycle, the bicycle, and an underwater vehicle.
\end{abstract}

\section{INTRODUCTION}

A bicycle, a car, an aircraft, or a submarine are but a few examples of nonholonomic systems. Planning trajectories for such systems is difficult because, by nature, some of their degrees of freedom can only be controlled in a coupled manner (see e.g. [9] and references therein). As a consequence, when such systems encounter on their ways an unexpected event (e.g. a random perturbation of the system state or of the target state, an unforeseen obstacle, etc.), it may be more efficient to deform in some manner the initially planned trajectory rather than to re-plan entirely a new one [7, 6, 8, 12].

Lamiraux and colleagues [8] suggested to iteratively deform the original path by perturbing infinitesimally the control inputs at each iteration. As underlined by Seiler and colleagues [12], that method requires re-integrating the whole trajectory at each iteration, which is computationally expensive. These authors then described a new method based on Lie group symmetries, which requires re-integrating only parts of the trajectory.

The Lie groups considered in [3, 12] are in fact Euclidean (or isometry) groups. We propose here to use larger Lie groups, namely, affine groups, which contain the Euclidean transformations as subgroups. Using affine transformations allows making more versatile trajectory corrections. In particular, the corrections are exact and can be computed algebraically, in one step, which makes iterative deformations [8] or gradient search [3, 12] unnecessary. Furthermore, there is no need to re-integrate even a part of the trajectory. Note that, in contrast with previous works where the studied systems are invariant under Euclidean transformations [3, 12], here trajectories and control inputs are not in general affine-invariant. More technical precautions need therefore to be taken to define and guarantee the feasibility (or admissibility) of the deformed trajectories under the system nonholonomic constraints. In particular, the admissibility conditions are formulated using differential equations with discontinuous right-hand sides [4].

In section II, we present the general framework of affine trajectory correction. We apply this framework, in sections III and $\mathbb{\nabla}$, to three classical examples in nonholonomic mobile robotics: the unicycle, the bicycle (or kinematic car), and an underwater vehicle. We discuss, in section $\nabla$, the advantages and drawbacks of the presented method, its domain of applicability, and possible future developments.

\section{AFFINE TRAJECTORY CORRECTION: GENERAL FRAMEWORK}

\section{A. Affine spaces and affine transformations}

An affine space is a set $\mathbb{A}$ together with a group action of a vector space $\mathbb{W}$. An element $\mathbf{w} \in \mathbb{W}$ transforms a point $P \in \mathbb{A}$ into another point $P^{\prime}$ by $P^{\prime}=P+\mathbf{w}$, which can also be noted $\overrightarrow{P P^{\prime}}=\mathbf{w}$.

Given a point $O \in \mathbb{A}$ (the origin), an affine transformation $\mathcal{F}$ of the affine space can be defined by a couple $(\mathbf{w}, \mathcal{M})$ where $\mathbf{w} \in \mathbb{W}$ and $\mathcal{M}$ is a non-singular endomorphism of $\mathbb{W}$ (i.e. a non-singular linear application $\mathbb{W} \rightarrow \mathbb{W}$ ). The transformation $\mathcal{F}$ acts on $\mathbb{A}$ by

$$
\forall P \in \mathbb{A} \quad \mathcal{F}(P)=O+\mathcal{M}(\overrightarrow{O P})+\mathbf{w} .
$$

Note that, if $P_{0}$ is a fixed-point of $\mathcal{F}$, then $\mathcal{F}$ can be written in the form

$$
\forall P \in \mathbb{A} \quad \mathcal{F}(P)=P_{0}+\mathcal{M}\left(\overrightarrow{P_{0} P}\right) .
$$

B. Admissible trajectories and admissible trajectory deformations

Let us consider a commanded system of dimension $N$. Suppose that $n$ of the system variables form an affine space. 
As an example, consider the unicycle model [9]

$$
\left\{\begin{array}{ccc}
\dot{v} & = & a \\
\dot{\theta} & = & \omega \\
\dot{x} & = & v \cos (\theta) \\
\dot{y} & = & v \sin (\theta)
\end{array},\right.
$$

where $(a, \omega)$ are the system control inputs (or commands) and $(x, y, \theta, v)$, the system variables. The $(x, y)$ space can be viewed as an affine space of dimension $n=2$. We call $(x, y)$ the base variables and the associated affine space, the base space.

We say that a full-space trajectory $\overline{\mathcal{C}}(t)_{t \in[0, T]}(\overline{\mathcal{C}}(t)=$ $(x(t), y(t), \theta(t), v(t))$ in the above example) is admissible if one can find a set of admissible commands ( $a$ and $\omega$ in the example) that generates $\overline{\mathcal{C}}$. A base-space trajectory $\mathcal{C}$ $(\mathcal{C}=(x, y)$ in the example) is admissible if there exists an admissible full-space trajectory whose projection on the base space coincides with $\mathcal{C}$.

Let $\mathcal{C}(t)_{t \in[0, T]}$ be a base-space trajectory and $\tau \in[0, T]$, a given time instant. We say that a transformation $\mathcal{F}$ occurring at $\tau$ deforms $\mathcal{C}(t)_{t \in[0, T]}$ into $\mathcal{C}^{\prime}(t)_{t \in[0, T]}$ if

$$
\begin{array}{ll}
\forall t<\tau & \mathcal{C}^{\prime}(t)=\mathcal{C}(t) \\
\forall t \geq \tau & \mathcal{C}^{\prime}(t)=\mathcal{F}(\mathcal{C}(t))
\end{array}
$$

Given an admissible base-space trajectory $\mathcal{C}$, an affine transformation $\mathcal{F}$ is said to be admissible if $\mathcal{F}$ deforms $\mathcal{C}$ into an admissible trajectory.

\section{Differential equations with discontinuous right-hand sides}

Throughout this manuscript, we assume that the admissible control inputs are piecewise continuous functions with finite limits at the discontinuity points - or piecewise $C^{0}$ functions. For convenience, we denote by $\mathscr{D}^{0}$ the space of such functions (see Fig. 四, top plot, for an example). Note that allowing discontinuities of the inputs is realistic. For instance, a car running on a straight road can be modeled by a system whose control input is the acceleration. In such a model, any brusque press on the throttle or on the brake pedal would correspond to a discontinuity of the acceleration input.

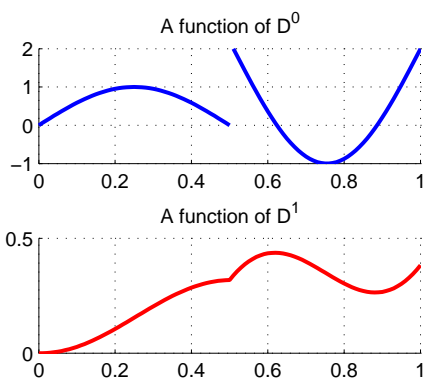

Fig. 1. Examples of functions of $\mathscr{D}^{0}$ (top) and of $\mathscr{D}^{1}$ (bottom). Note that the top function is actually the derivative of the bottom function.

Let $u$ be a function of $\mathscr{D}^{0}$ and consider the following dif- ferential equation with discontinuous right-hand side (see [4])

$$
\left\{\begin{array}{l}
\dot{x}(t)=u(t) \\
x(0)=x_{0}
\end{array} .\right.
$$

Any solution $x$ of system (ম) is continuous and, from the definition of $\mathscr{D}^{0}$, piecewise $C^{1}$. Conversely, given any function $x$ that is $C^{0}$ and piecewise $C^{1}$, one has $\dot{x} \in \mathscr{D}^{0}$. For convenience, we denote by $\mathscr{D}^{1}$ the space of such $x$ functions

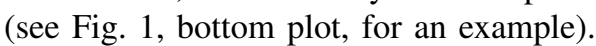

Finally, we denote by $\mathscr{D}^{2}$ the space of differentiable functions whose derivatives are in $\mathscr{D}^{1}$. This definition does not involve technical difficulties since the functions of $\mathscr{D}^{1}$ are continuous.

Typically, for a system commanded in acceleration, the acceleration $a$ would belong to $\mathscr{D}^{0}$, the velocity $v$ to $\mathscr{D}^{1}$, and the position $(x, y)$ to $\mathscr{D}^{2}$.

\section{Dimension of the space of admissible affine deformations}

From the previous section, one can see that, typically, some of the system variables are required to be continuous. These continuity conditions are particularly critical at the time instant $\tau$ when the deformation occurs. In general, if one needs to guarantee the continuities of $m$ system variables at $\tau$, this will define $m$ constraints on the set of admissible affine transformations. On the other hand, the affine transformations of an $n$-dimensional space form the Lie group $\mathrm{GA}_{n}$ of dimension $n+n^{2}$ ( $n$ coordinates for the translation and $n^{2}$ coordinates for the endomorphism of the associated vector space, where $n$ is the number of base variables). Consequently, if $n+n^{2}>m$, we could expect to have at our disposal $\tau$ and $n+n^{2}-m$ "extra degrees of freedom" to achieve the desired correction while staying admissible.

For the unicycle (section IIII) and the bicycle (section $\mathbb{D - A}$ ), the base space $(x, y)$ is of dimension $n=2$. We show that there are respectively $m=4$ and $m=5$ continuity conditions for these systems, yielding respectively $n+n^{2}-m=2$ and $n+n^{2}-m=1$ "extra degrees of freedom". We then suggest how to play with $\tau$ and these "extra degrees of freedom" to make corrections towards virtually any desired final position and orientation. For the underwater vehicle (section $\mathbb{D V - B})$, the base space $(x, y, z)$ is of dimension $n=3$ and there are $m=6$ continuity conditions, yielding $n+n^{2}-m=6$ "extra degrees of freedom".

\section{AFFINE TRAJECTORY CORRECTION: ILLUSTRATION}

The above presented framework suggests the following general scheme to study affine trajectory correction for a particular system

1) check the conditions for a base-space trajectory to be admissible;

2) characterize the set of admissible affine deformations;

3) compute the admissible affine deformation that achieves the desired trajectory correction.

To illustrate, let us now apply the above scheme to a simple classical nonholonomic system: the unicycle. 


\section{A. Admissible trajectories}

As mentioned previously, a unicycle can be modeled by system (II), where the admissible inputs $a$ and $\omega$ are in $\mathscr{D}^{0}$ (see section एᄑ-C). From the system equations, it is clear that, for any admissible full-space trajectory of the unicycle, $v$ and $\theta$ must be in $\mathscr{D}^{1}$ and $x$ and $y$ must be in $\mathscr{D}^{2}$.

Conversely, given a base-space trajectory $\mathcal{C}(t)_{t \in[0, T]}=$ $(x(t), y(t))_{t \in[0, T]}$ that is in $\mathscr{D}^{2}$, one can safely write

$$
\left\{\begin{array}{cccc}
\theta & = & \arctan 2(\dot{y}, \dot{x}) \\
v & = & \sqrt{\dot{x}^{2}+\dot{y}^{2}} \\
a & = & \dot{v} \\
\omega & = & \dot{\theta}
\end{array},\right.
$$

which allows computing back the non-base variables $v$ and $\theta$ (in $\mathscr{D}^{1}$ ) as well as the control inputs $a$ and $\omega$ (in $\mathscr{D}^{0}$ ). This in turn implies the admissibility of $\mathcal{C}(t)_{t \in[0, T]}$. In summary, a base-space trajectory is admissible if and only if it belongs to $\mathscr{D}^{2}$.

Important remark: From a computational viewpoint, equations (B3) suggest that, if one obtains an admissible basespace trajectory $\mathcal{C}^{\prime}(t)_{t \in[0, T]}$ (for instance by deforming a given $\mathcal{C}(t)_{t \in[0, T]}$, it will be easy to compute the control inputs that generate that trajectory by some differentiations and elementary operations. $\triangle$

\section{B. Admissible affine deformations}

Consider now an admissible base-space trajectory $\mathcal{C}(t)_{t \in[0, T]}$ and an affine deformation $\mathcal{F}$ occurring at time $\tau$. One has by definition $\mathcal{C}^{\prime}(t)_{t \in(\tau, T]}=\mathcal{F}\left(\mathcal{C}(t)_{t \in(\tau, T]}\right)$. Since $\mathcal{F}$ is a smooth application, it is clear that $\mathcal{C}^{\prime}(t)_{t \in(\tau, T]}$ - note that the interval is open at $\tau$ - is in $\mathscr{D}^{2}$ if and only if $\mathcal{C}(t)_{t \in(\tau, T]}$ is in $\mathscr{D}^{2}$.

Regarding the time instant $\tau$, the continuities of $x$ and $y$ impose that $\mathcal{F}(\mathcal{C}(\tau))=\mathcal{C}(\tau)$. Thus $\mathcal{F}$ can be written in the form

$$
\forall P \in \mathbb{A} \quad \mathcal{F}(P)=\mathcal{C}(\tau)+\mathcal{M}(\overrightarrow{\mathcal{C}(\tau) P}) .
$$

One now needs to guarantee the continuities of $v$ and $\theta$ at $\tau$, since the two remaining conditions (differentiability and finite limits for the derivative) do not depend on the behavior of $\mathcal{C}^{\prime}$ at the discrete point $\tau$, and are therefore already satisfied by virtue of the smoothness of $\mathcal{F}$.

Consider for this the velocity vector $\mathbf{v}=(\dot{x}, \dot{y})^{\top}$. Remark that the continuity of this velocity vector is equivalent to that of $v$ and $\theta$. The continuities of $\mathbf{v}$ mean that $\mathbf{v}(\tau-)$ and $\mathbf{v}(\tau+)$ (where the signs - and + denote respectively the left and right limits) are well defined, and that $\mathbf{v}(\tau-)=\mathbf{v}(\tau+)=\mathbf{v}(\tau)$.

Similarly, the continuity of $\mathbf{v}^{\prime}$ would mean that $\mathbf{v}^{\prime}(\tau+)=$ $\mathbf{v}^{\prime}(\tau-)=\mathbf{v}(\tau)$. On the other hand, one has $\mathbf{v}^{\prime}(\tau+)=$ $\mathcal{M}(\mathbf{v}(\tau))$. These equalities together imply $\mathcal{M}(\mathbf{v}(\tau))=\mathbf{v}(\tau)$.

Let us now decompose $\mathcal{M}$ is the basis $\left\{\mathbf{u}_{\|}, \mathbf{u}_{\perp}\right\}$ where $\mathbf{u}_{\|}=(\cos (\theta), \sin (\theta))$ is the unit tangent vector and $\mathbf{u}_{\perp}=$ $(-\sin (\theta), \cos (\theta))$ is the unit normal vector. The condition $\mathcal{M}(\mathbf{v}(\tau))=\mathbf{v}(\tau)$ is equivalent to

$$
\exists \lambda, \mu \in \mathbb{R} \quad \mathbf{M}=\left(\begin{array}{cc}
1 & \lambda \\
0 & 1+\mu
\end{array}\right)
$$

where $\mathbf{M}$ is the matrix representing $\mathcal{M}$ in the basis $\left\{\mathbf{u}_{\|}, \mathbf{u}_{\perp}\right\}$.

In summary (and in relation with the discussion of section (II-D), the admissible affine transformations at time $\tau$ form a Lie subgroup of dimension 2 of $\mathrm{GA}_{2}$, parameterized by $\lambda$ and $\mu$ in equation (Б).

Physical constraints: In practice, other constraints, such as upper limits on the absolute acceleration or on the trajectory curvature, further restrict the set of admissible affine deformations. This can also be treated in the proposed framework, since the changes in acceleration or curvature from the original trajectory can be easily computed from the affine transformation at hand (see also []] $). \triangle$

\section{Trajectory correction}

We consider only the correction of the final position and assume that $\tau$ is given. It is possible to achieve more complex corrections (e.g. correcting the final orientation) or to choose "optimal" $\tau \mathrm{s}$ : these developments are left to the reader.

From equation (4), to correct the final position $\mathcal{C}(T)$ towards a desired position $P_{d}=\left(x_{d}, y_{d}\right)$, one needs to look for a linear application $\mathcal{M}$ such that

$$
\mathcal{M}(\overrightarrow{\mathcal{C}(\tau) \mathcal{C}(T)})=\overrightarrow{\mathcal{C}(\tau) P_{d}}
$$

Let $\mathbf{Q}=\left[\mathbf{u}_{\|}, \mathbf{u}_{\perp}\right]$ and let the matrix representing $\mathcal{M}$ in the basis $\left\{\mathbf{u}_{\|}, \mathbf{u}_{\perp}\right\}$ be

$$
\mathbf{M}=\left(\begin{array}{cc}
1 & \lambda \\
0 & 1+\mu
\end{array}\right)
$$

Equation (6) implies

$$
\mathbf{Q M Q}^{-1}\left(\begin{array}{c}
x(T)-x(\tau) \\
y(T)-y(\tau)
\end{array}\right)=\left(\begin{array}{c}
x_{d}-x(\tau) \\
y_{d}-y(\tau)
\end{array}\right) .
$$

Let next

$$
\begin{gathered}
\left(x_{1}, y_{1}\right)^{\top}=\mathbf{Q}^{-1}(x(T)-x(\tau), y(T)-y(\tau))^{\top} \\
\left(x_{2}, y_{2}\right)^{\top}=\mathbf{Q}^{-1}\left(x_{d}-x(\tau), y_{d}-y(\tau)\right)^{\top} .
\end{gathered}
$$

Equation (पD) then implies

$$
\lambda=\left(x_{2}-x_{1}\right) / y_{1}, \quad \mu=\left(y_{2}-y_{1}\right) / y_{1},
$$

provided that $y_{1} \neq 0$, i.e. that the tangent at $\tau$ does not go through $\mathcal{C}(T)$. Fig. \ shows examples of trajectory corrections for the unicycle.

Note that any desired position in the whole space - and not only those in the vicinity of the initially planned final position as in [12] - can theoretically be reached. Remark on the other hand that the distance (e.g. the $L_{2}$ distance) of the corrected trajectory from the original trajectory is a continuous function of $\lambda$ and $\mu$, meaning that using small $\lambda \mathrm{s}$ and $\mu$ s results in small changes in the overall trajectory (and in the commands).

\section{Obstacle avoidance, meeting trajectory constraints}

In the previous development, one can in fact replace $T$ by any time instant $t>\tau$. This allows implementing interactive obstacle avoidance algorithms as follows (see Fig. \ for an example) 
1) determine a time instant $t_{\text {obs }}$ when the initially planned trajectory would collide with the obstacle;

2) select a new, non colliding, intermediate position $\left(x_{\text {inter }}, y_{\text {inter }}\right)$ to which one could make a correction;

3) make the correction of $\left(x\left(t_{\mathrm{obs}}\right), y\left(t_{\mathrm{obs}}\right)\right)$ towards $\left(x_{\text {inter }}, y_{\text {inter }}\right)$, with a $\tau_{1}<t_{\text {obs }}$;

4) re-correct the final position towards the initially planned final position, with a $\tau_{2} \geq t_{\mathrm{obs}}$.
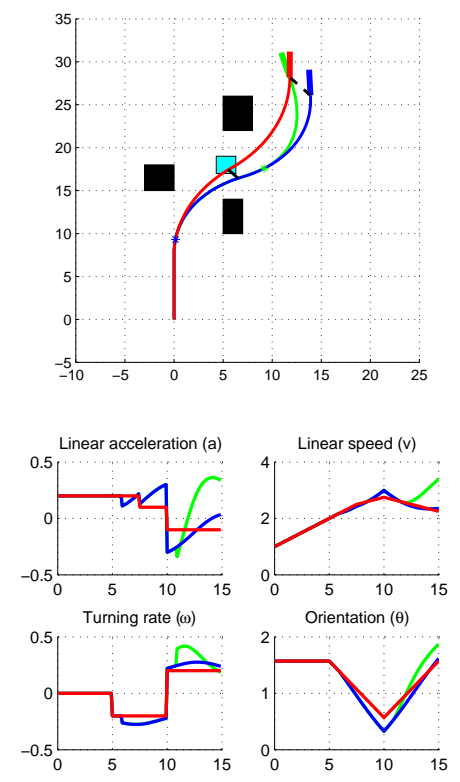

Fig. 2. An example of obstacle avoidance for the unicycle. The original trajectory $(x, y)$ (red) was planned knowing the position of the black obstacles. During the execution, an unforeseen obstacle (cyan) appears on the original path. A new trajectory $\left(x_{1}, y_{1}\right)$ (blue) is obtained by deforming the original trajectory. The blue star indicates the position $\left(x\left(\tau_{1}\right), y\left(\tau_{1}\right)\right)$ where the deformation occurs, and the black plain line joins $\left(x\left(t_{\mathrm{obs}}\right), y\left(t_{\mathrm{obs}}\right)\right)$ to $\left(x_{\text {inter }}, y_{\text {inter }}\right)$. Next, in order to get back to the original target, an other trajectory (green) is obtained by deforming the blue one. The green star indicates the position $\left(x_{1}\left(\tau_{2}\right), y_{1}\left(\tau_{2}\right)\right)$ where the deformation occurs, and the black dashed line joins $\left(x_{1}(T), y_{1}(T)\right)$ to $(x(T), y(T))$.

This algorithm can be run iteratively to avoid all obstacles.

One can also prescribe a specific position/orientation of the trajectory at a given time instant $t_{\text {door }}$ (this is desirable for instance when two large obstacles are close to each other, leaving between them a small doorway through which the robot can pass), as follows

1) make the correction of $\left(x\left(t_{\text {door }}\right), y\left(t_{\text {door }}\right)\right)$ towards the specified intermediate position;

2) make the correction of $\theta\left(t_{\text {door }}\right)$ towards the specified intermediate orientation;

3) re-correct the final position towards the initially planned final position, using a $\tau>t_{\text {door }}$.

\section{OTHER EXAMPLES}

To further demonstrate the possibilities offered by affine trajectory deformations, we now briefly discuss two more complex cases: the bicycle (section $[\nabla-A)$, which can be viewed as a unicycle whose angular speed is required to be continuous, and an underwater vehicle (section [V-B), which is a natural $3 \mathrm{D}$ extension of the unicycle. This section can be skipped on the first reading.

\section{A. The bicycle}

A bicycle (or kinematic car) can be modeled by the following system [9]

$$
\left\{\begin{array}{lcc}
\dot{v} & = & a \\
\dot{\phi} & = & \rho \\
\dot{\theta} & = & \frac{v \tan (\phi)}{L} \\
\dot{x} & = & v \cos (\theta) \\
\dot{y} & = & v \sin (\theta)
\end{array},\right.
$$

where $(a, \rho)$ are the system control inputs and $\overline{\mathcal{C}}=$ $(x, y, \theta, v, \phi)$, the system variables.

1) Admissible trajectories: Consider a base-space trajectory $\mathcal{C}=(x, y) \in \mathscr{D}^{2}$. As in section WI-B, equations (B) allow computing $v \in \mathscr{D}^{1}, a \in \mathscr{D}^{0}$ and $\theta \in \mathscr{D}^{1}$. Next, if $v$ is always nonzero, one can compute $\phi$ by

$$
\phi=\arctan (\dot{\theta} L / v) \text {. }
$$

Since $\theta \in \mathscr{D}^{1}$, equation (8) implies that $\phi \in \mathscr{D}^{0}$, but not necessarily that $\phi \in \mathscr{D}^{1}$. Next, one can compute $\rho=\dot{\phi}$. For $\rho$ to be in $\mathscr{D}^{0}$, one would need $\phi \in \mathscr{D}^{1}$. But as just remarked, the latter condition is not automatically guaranteed by $\mathcal{C}=$ $(x, y) \in \mathscr{D}^{2}$. On the other hand, demanding that $\mathcal{C}=(x, y) \in$ $\mathscr{D}^{3}$ would be unduly restrictive. Thus the condition $\phi \in \mathscr{D}^{1}$ must be specified as an independent supplementary condition. In summary, if $\mathcal{C}=(x, y) \in \mathscr{D}^{2}$ and if $\phi-$ as computed from $\mathcal{C}$ by equations $(\mathbb{B})$ and $(\mathbb{B})-$ belongs to $\mathscr{D}^{1}$, then $\mathcal{C}$ is admissible. Conversely, if $\mathcal{C}=(x, y)$ is admissible, one can easily show that $(x, y) \in \mathscr{D}^{2}$ and $\phi \in \mathscr{D}^{1}$.

Note that these admissibility conditions can also be formulated in terms of continuity constraints on the path curvature [2, 5].

2) Admissible affine deformations: Consider now an admissible base-space trajectory $\mathcal{C}$ and an affine deformation $\mathcal{F}$ occurring at time $\tau$ that deforms $\mathcal{C}$ into $\mathcal{C}^{\prime}$. As in section $\mathrm{II-B}$, one can show that $\mathcal{C}^{\prime}(t)_{t \in(\tau, T]}$ is in $\mathscr{D}^{2}$ if and only if $\mathcal{F}(\mathbf{v}(\tau))=\mathbf{v}(\tau)$, where $\mathbf{v}$ is the velocity vector. One now needs to check the continuities of $\phi^{\prime}$ at $\tau$ and at the discontinuity points of the second derivative of $\mathcal{C}$ (the continuity and differentiability of $\phi^{\prime}$ elsewhere are already guaranteed by the smoothness of $\mathcal{F}$, cf. section [II-B).

Consider for this the acceleration vector $\mathbf{a}=(\ddot{x}, \ddot{y})^{\top}$. By definition, one has

$$
\mathbf{a}=a \mathbf{u}_{\|}+\frac{v^{2} \tan (\phi)}{L} \mathbf{u}_{\perp}
$$

with a not necessarily continuous. One can next write

$$
\mathbf{a} \cdot \mathbf{u}_{\perp}=\frac{v^{2} \tan (\phi)}{L} .
$$

Consider now a time instant $t>\tau$ when $\mathbf{a}$ is possibly discontinuous, that is $\mathbf{a}(t-) \neq \mathbf{a}(t+)$. Since $\phi$ and $v$ are continuous, one has by equation (Q)

$$
\mathbf{a}(t-) \cdot \mathbf{u}_{\perp}(t)=\mathbf{a}(t+) \cdot \mathbf{u}_{\perp}(t),
$$


or, in other words, that $\mathbf{a}(t+)-\mathbf{a}(t-)$ and $\mathbf{u}_{\|}(t)$ are collinear. Here comes into play a nice property of affine transformations: they preserve collinearity. Using this property, one obtains that $\mathcal{M}(\mathbf{a}(t+)-\mathbf{a}(t-))$ and $\mathcal{M}\left(\mathbf{u}_{\|}(t)\right)$ are collinear. But the former vector is no other than $\mathbf{a}^{\prime}(t+)-\mathbf{a}^{\prime}(t-)$ and the latter is collinear with $\mathbf{u}_{\|}^{\prime}(t)$, since

$$
\mathbf{u}_{\|}^{\prime}(t)=\frac{\mathcal{M}\left(\mathbf{u}_{\|}(t)\right)}{\left\|\mathcal{M}\left(\mathbf{u}_{\|}(t)\right)\right\|} .
$$

Thus $\mathbf{a}^{\prime}(t-) \cdot \mathbf{u}_{\perp}^{\prime}(t)=\mathbf{a}^{\prime}(t+) \cdot \mathbf{u}_{\perp}^{\prime}(t)$, which in turn implies the continuity of $\phi^{\prime}$ at $t$ (note that this conclusion also relies on the fact that $v^{\prime}$ is nonzero if $v$ is nonzero, owing to the non-singularity of $\mathcal{M}$ ).

Remark: Since the affine group is the largest transformation group of the plane that preserves collinearity, the previous development shows that it is also the largest group that preserves the admissibility of every bicycle trajectory! $\triangle$

Turning now to the time instant $\tau$, the same reasoning as previously shows that $\phi^{\prime}$ is continuous at $\tau$ if and only if

$$
\mathbf{a}^{\prime}(\tau+) \cdot \mathbf{u}_{\perp}(\tau)=\mathbf{a}(\tau) \cdot \mathbf{u}_{\perp}(\tau),
$$

or equivalently, if

$$
\mathcal{M}(\mathbf{a}(\tau)) \cdot \mathbf{u}_{\perp}(\tau)=\mathbf{a}(\tau) \cdot \mathbf{u}_{\perp}(\tau) .
$$

Remark now that, since $\mathbf{v} \cdot \mathbf{u}_{\perp}=0$, condition (ए0) is in fact equivalent to

$$
\exists \lambda \in \mathbb{R} \quad \mathcal{M}(\mathbf{a}(\tau))=\mathbf{a}(\tau)+\lambda \mathbf{v}(\tau) .
$$

Denoting by $\mathcal{B}$ the application such that $\mathcal{B}(\mathbf{v}(\tau))=\mathbf{0}$ and $\mathcal{B}(\mathbf{a}(\tau))=\mathbf{v}(\tau)$ (one can compute $\mathcal{B}$ explicitly by $\left.\mathcal{B}=[\mathbf{0}, \mathbf{v}(\tau)][\mathbf{v}(\tau), \mathbf{a}(\tau)]^{-1}\right)$, one obtains

$$
\exists \lambda \in \mathbb{R} \quad \mathcal{M}=\mathcal{I}+\lambda \mathcal{B} .
$$

In summary (and in relation with the discussion of section (II-D), the admissible affine transformations at time $\tau$ form a Lie subgroup of dimension 1 of $\mathrm{GA}_{2}$, given by $\{\mathcal{I}+\lambda \mathcal{B}\}_{\lambda \in \mathbb{R}}$.

Inflection points: The previous development is valid only when $\mathbf{v}(\tau)$ and $\mathbf{a}(\tau)$ are not collinear, that is, when $\mathcal{C}(\tau)$ is not an inflection point (see also [I] for an interesting discussion on inflection points in the context of human movements). $\triangle$

3) Trajectory correction: Let us now play with $\tau$ and the "extra degree of freedom" $\lambda$ to make trajectory corrections.

For this, we first study how the final position of the trajectory $\mathcal{C}(T)$ is affected by an admissible affine deformation occurring at time $\tau$. By definition, one has

$$
\begin{aligned}
\mathcal{C}^{\prime}(T) & =\mathcal{C}(\tau)+(\mathcal{I}+\lambda \mathcal{B})(\overrightarrow{\mathcal{C}(\tau) \mathcal{C}(T)}) \\
& =\mathcal{C}(T)+\lambda \mathcal{B}(\overrightarrow{\mathcal{C}(\tau) \mathcal{C}(T)})
\end{aligned}
$$

Let us decompose $\overrightarrow{\mathcal{C}(\tau) \mathcal{C}(T)}$ in the (in general nonorthonormal) basis $\{\mathbf{v}(\tau), \mathbf{a}(\tau)\}$

$$
\overrightarrow{\mathcal{C}(\tau) \mathcal{C}(T)}=\alpha \mathbf{v}(\tau)+\beta \mathbf{a}(\tau)
$$

By definition of $\mathcal{B}$, one has

$$
\mathcal{C}^{\prime}(T)=\mathcal{C}(T)+\lambda \beta \mathbf{v}(\tau) .
$$

Consequently, if $\beta$ is nonzero (that is, if $\overrightarrow{\mathcal{C}(\tau) \mathcal{C}(T)}$ and $\mathbf{v}(\tau)$ are non-collinear, or in other words, if the tangent at $\tau$ does not go through $\mathcal{C}(T)$ ), then the locus of $\mathcal{C}^{\prime}(T)$ when $\lambda$ varies is the line that goes through $\mathcal{C}(T)$ and that is collinear with $\mathbf{v}(\tau)$.

In order to make a correction of the final position from $\mathcal{C}(T)$ to a desired position $P_{d}$, it therefore suffices to (see Fig. B)

1) compute the vector $\mathbf{e}_{d}=\overrightarrow{\mathcal{C}(T) P_{d}}$;

2) find a time instant $\tau$ when the tangent $\mathbf{u}_{\|}(\tau)$ is collinear with $\mathbf{e}_{d}$;

3) compute $\lambda=\overline{\mathbf{e}_{d}} /(\beta \overline{\mathbf{v}(\tau)})$ where the overline denotes the signed norm;

4) make the affine deformation of parameter $\lambda$ at time $\tau$.
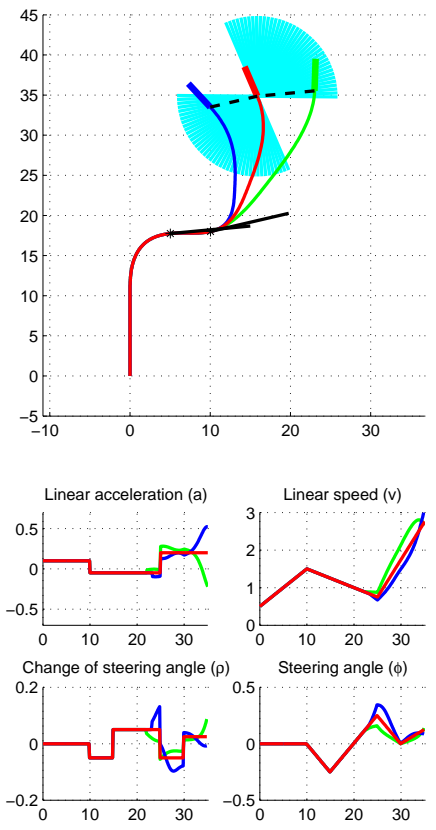

Fig. 3. Accessible final positions (in cyan) and two examples of position corrections. The original trajectory is in red. For each correction, the black plain line represents the tangent at $\tau$ while the black dotted line joins the original final position $C(T)$ to the desired final position $P_{d}$. Note the collinearity of the plain line and the dotted line.

More complex corrections can be achieved by choosing appropriate $\tau \mathrm{s}$ : we provide for instance in the Appendix an algorithm to correct the final orientation.

Accessible positions: From the previous development, it appears that a position $P_{d}$ is accessible if and only if the original trajectory $\mathcal{C}(t)_{t \in[0, T]}$ has a tangent that is parallel to $\overrightarrow{\mathcal{C}(T) P_{d}}$. Therefore the set of the trajectory tangents (minus the tangents at the inflection points) determine the accessible directions for position corrections, as shown in Fig. B]. In practical terms, the more orientations the bicycle experiences during its initially planned movement, the more positions can be reached trough affine deformations. During the initial planning stage, there will therefore be a trade-off between "optimizing" the trajectory (e.g. minimizing the path length or the norms of the control inputs) and having the trajectory make 
many detours (providing thereby more tangents to deal with unexpected events). Note that composing several deformations allows bypassing these restrictions. $\triangle$

\section{B. An underwater vehicle}

An underwater vehicle can be modeled by the following system [11]

$$
\left\{\begin{array}{ccc}
\dot{v} & = & a \\
\left(\begin{array}{c}
\dot{\phi} \\
\dot{\theta} \\
\dot{\psi}
\end{array}\right) & = & \mathbf{R}(\phi, \theta)\left(\begin{array}{c}
\omega_{x} \\
\omega_{y} \\
\omega_{z}
\end{array}\right), \\
\dot{x} & = & v \cos \psi \cos \theta \\
\dot{y} & = & v \sin \psi \cos \theta \\
\dot{z} & = & -v \sin \theta
\end{array}\right.
$$

where $\left(a, \omega_{x}, \omega_{y}, \omega_{z}\right)$ are the system control inputs, $\overline{\mathcal{C}}=$ $(x, y, z, \phi, \theta, \psi, v)$, the system variables, and

$$
\mathbf{R}(\phi, \theta)=\left(\begin{array}{ccc}
1 & \sin \phi \tan \theta & \cos \phi \tan \theta \\
0 & \cos \phi & -\sin \phi \\
0 & \sin \phi \sec \theta & \cos \phi \sec \theta
\end{array}\right) .
$$

1) Admissible trajectories: Following the same line of reasoning as previously, a necessary condition for the admissibility of a base-space trajectory $\mathcal{C}(t)_{t \in[0, T]}=$ $(x(t), y(t), z(t))_{t \in[0, T]}$ is that $x, y$ and $z$ belong to $\mathscr{D}^{2}$.

Conversely, assume that $x, y$ and $z$ belong to $\mathscr{D}^{2}$. Remark first that, from the system's equations (12), the "roll" angle $\phi$ is independent of $(x(t), y(t), z(t))_{t \in[0, T]}$. Next, given an "extra" roll angle profile $\phi(t)_{t \in[0, T]} \in \mathscr{D}^{1}$, one can safely write (assuming that the velocity is always nonzero and that the trajectory stays away from the singularities of the Euler convention [1]])

$$
\left\{\begin{array}{ccc}
\psi & = & \arctan 2(\dot{y}, \dot{x}) \\
v & = & \sqrt{\dot{x}^{2}+\dot{y}^{2}+\dot{z}^{2}} \\
\theta & = & \arcsin (\dot{z} / v) \\
a & = & \dot{v} \\
\left(\begin{array}{c}
\omega_{x} \\
\omega_{y} \\
\omega_{z}
\end{array}\right)= & & \mathbf{R}(\phi, \theta)^{-1}\left(\begin{array}{c}
\dot{\phi} \\
\dot{\theta} \\
\dot{\psi}
\end{array}\right)
\end{array}\right.
$$

In summary, a base-space trajectory is admissible if and only if it is in $\mathscr{D}^{2}$.

2) Admissible affine deformations: Consider now an admissible base-space trajectory $\mathcal{C}$ and an affine deformation $\mathcal{F}$ occurring at time $\tau$ that deforms $\mathcal{C}$ in to $\mathcal{C}^{\prime}$. As in section III-B, one can show that $\mathcal{C}^{\prime}(t)_{t \in(\tau, T]}$ belongs to $\mathscr{D}^{2}$, owing to the smoothness of $\mathcal{F}$.

At the time instant $\tau$, the continuities of $x, y$ and $z$ impose that $\mathcal{F}(\mathcal{C}(\tau))=\mathcal{C}(\tau)$. Thus $\mathcal{F}$ can be written in the form

$$
\forall P \in \mathbb{A} \quad \mathcal{F}(P)=\mathcal{C}(\tau)+\mathcal{M}(\overrightarrow{\mathcal{C}(\tau) P}) .
$$

Next, following again the same reasoning as in section [1]-B, the continuities of $v, \psi$ and $\theta$ are equivalent to setting $\mathcal{M}(\mathbf{v}(\tau))=\mathbf{v}(\tau)$.

In summary, an affine deformation $\mathcal{F}$ occurring at time $\tau$ is admissible if and only if $\mathcal{M}(\mathbf{v}(\tau))=\mathbf{v}(\tau)$ when $\mathcal{F}$ is written in the form (113). As a consequence, the admissible affine transformations at time $\tau$ form a Lie subgroup of dimension 6 of $\mathrm{GA}_{3}$.

In practice, we shall compute $\mathcal{M}$ in the basis $\left\{\mathbf{u}_{\|}, \mathbf{w}_{1}, \mathbf{w}_{2}\right\}$ where $\mathbf{w}_{1}$ and $\mathbf{w}_{2}$ are two unit vectors forming an orthonormal basis with $\mathbf{u}_{\|}$. In this basis, the condition $\mathcal{M}(\mathbf{v}(\tau))=\mathbf{v}(\tau)$ is equivalent to setting the first column of the matrix that represents $\mathcal{M}$ to $(1,0,0)$. It suffices therefore to find the six remaining coefficients.

3) Trajectory correction: We consider only the correction of the final position, at a given $\tau$. It is possible to achieve more complex corrections as well (correcting the final orientation, avoiding obstacles, etc.) or to optimize the time instant $\tau$ : these developments are left to the reader.

Theoretically, three free coefficients are sufficient to reach any final position. As a consequence, we have here more coefficients than what is needed. We solve this "redundancy" problem by choosing an affine transformation that is the "closest" to the identity matrix, i.e., that affects the least the original trajectory.

As in section II-C, to correct towards a desired position $P_{d}=\left(x_{d}, y_{d}, z_{d}\right)$, one needs to look for a linear application $\mathcal{M}$ such that

$$
\mathcal{M}(\overrightarrow{\mathcal{C}(\tau) \mathcal{C}(T)})=\overrightarrow{\mathcal{C}(\tau) P_{d}}
$$

Let $\mathbf{Q}=\left[\mathbf{u}_{\|}, \mathbf{w}_{1}, \mathbf{w}_{2}\right]$ and let the matrix representing $\mathcal{M}$ in the basis $\left\{\mathbf{u}_{\|}, \mathbf{w}_{1}, \mathbf{w}_{2}\right\}$ be

$$
\mathbf{M}=\left(\begin{array}{ccc}
1 & \lambda & \mu \\
0 & 1+\nu & \xi \\
0 & \sigma & 1+\chi
\end{array}\right)
$$

Equation (펴) implies

$$
\mathbf{Q M Q}^{-1}\left(\begin{array}{c}
x(T)-x(\tau) \\
y(T)-y(\tau) \\
z(T)-z(\tau)
\end{array}\right)=\left(\begin{array}{c}
x_{d}-x(\tau) \\
y_{d}-y(\tau) \\
z_{d}-z(\tau)
\end{array}\right)
$$

Let next

$$
\begin{gathered}
\left(x_{1}, y_{1}, z_{1}\right)^{\top}=\mathbf{Q}^{-1}(x(T)-x(\tau), y(T)-y(\tau), z(T)-z(\tau))^{\top}, \\
\left(x_{2}, y_{2}, z_{2}\right)^{\top}=\mathbf{Q}^{-1}\left(x_{d}-x(\tau), y_{d}-y(\tau), z_{d}-z(\tau)\right)^{\top} .
\end{gathered}
$$

Equation ([15) then implies

$$
\mathbf{U}\left(\begin{array}{l}
\lambda \\
\mu \\
\nu \\
\xi \\
\sigma \\
\chi
\end{array}\right)=\left(\begin{array}{l}
x_{2}-x_{1} \\
y_{2}-y_{1} \\
z_{2}-z_{1}
\end{array}\right)
$$

where

$$
\mathbf{U}=\left(\begin{array}{cccccc}
y_{1} & z_{1} & 0 & 0 & 0 & 0 \\
0 & 0 & y_{1} & z_{1} & 0 & 0 \\
0 & 0 & 0 & 0 & y_{1} & z_{1}
\end{array}\right)
$$

The $(\lambda, \mu, \nu, \xi, \sigma, \chi)$ with minimal norm (i.e. that yields a $\mathcal{M}$ closest to identity according to the Frobenius distance) and that satisfies the under-determined system (ㅁ) is given by $\mathbf{U}^{+}\left(x_{2}-x_{1}, y_{2}-y_{1}, z_{2}-z_{1}\right)^{\top}$, where $\mathbf{U}^{+}$denotes the 
Moore-Penrose pseudo-inverse of $\mathbf{U}$.

Finally, one needs to choose the "independent" angle $\phi(t)_{t \in[\tau, T]}$. Here our strategy consists of keeping the same $\phi$ as in the original trajectory. Other strategies (e.g. keeping the same absolute roll as in the original trajectory) can also be used. Fig. 目 shows some examples of trajectory corrections.
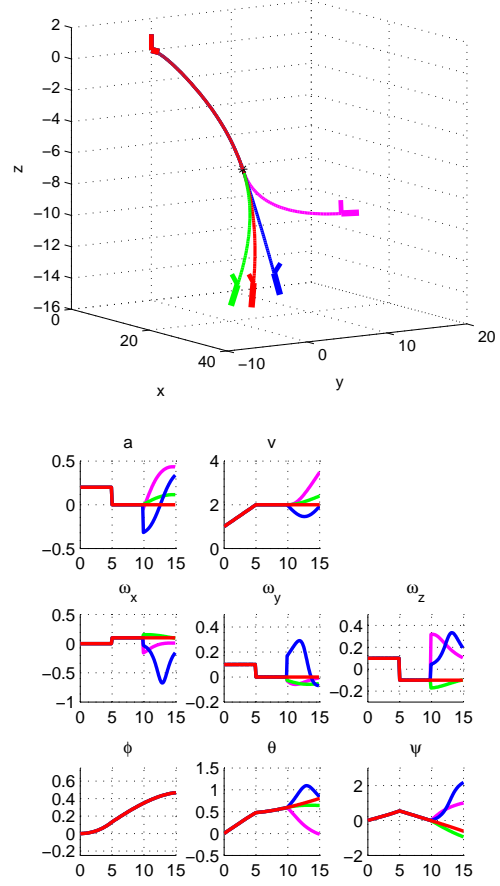

Fig. 4. Examples of trajectory corrections for an underwater vehicle. The original trajectory is in red.

\section{DISCUSSION}

As stated at the beginning of section III, one can apply the following general scheme to study affine trajectory corrections for nonholonomic systems

1) check the conditions for a base-space trajectory to be admissible. Often (but not always), a base-space trajectory is admissible if it - and some functions computed from it - belong to certain classes $\mathscr{D}^{i}$;

2) based on the admissibility conditions of trajectories, particularly at the time instant when the deformation occurs, characterize the set of admissible affine deformations. Often (but not always), the admissible affine deformations at a given time instant form a Lie group of dimension $n+n^{2}-m$ where $n$ is the number of base variables and $m$ the number of continuity conditions;

3) finally, play with $\tau$ and the $n+n^{2}-m$ "extra degrees of freedom" to achieve the desired correction. If there are more "extra degrees of freedom" than needed, one can "optimize" by choosing the affine transformations that are the closest to identity.

This general scheme suggests in turn the classes of systems that can or cannot be tackled by the proposed method. For instance, an underwater vehicle whose changes in turning rate ( $\rho_{x}=\dot{\omega}_{x}, \rho_{y}=\dot{\omega}_{y}, \rho_{z}=\dot{\omega}_{z}$ ) are commanded instead of the turning rates $\left(\omega_{x}, \omega_{y}, \omega_{z}\right)$ could probably be treated by the method (since in this case $n+n^{2}-m=3$ ). The development of the theory to deal with general classes of nonholonomic systems are also the subject of ongoing efforts.

As mentioned in the Introduction, one advantage of the proposed method is that it requires no re-integration of the trajectory. On the other hand, differentiations of the trajectory must be performed in order to recover the commands (see "Important remark" in section (II-A). Note however that, if multiple deformations are made (for instance to avoid multiple obstacles), the differentiations need to be performed only once, after all the deformations have been applied.

The group property of affine transformations can also be used to further accelerate the computations (as in [12] with Euclidean transformations). Assume for instance that two affine transformations $\mathcal{F}_{1}$ and $\mathcal{F}_{2}$ are applied at time instants $\tau_{1}$ and $\tau_{2}$, with $\tau_{1}<\tau_{2}$. Then one can apply $\mathcal{F}_{1}$ to $\mathcal{C}(t)_{t \in\left[\tau_{1}, \tau_{2}\right]}$ and next $\mathcal{F}_{2} \circ \mathcal{F}_{1}$, which is also an affine transformation, to $\mathcal{C}(t)_{t \in\left(\tau_{2}, T\right]}$.

Another advantage, also mentioned in the Introduction, is that our method can be executed in one step, while other methods require iterative deformations of the trajectory [8] or gradient descent to find the appropriate deformation coefficients [3, 12]. This may result in significant performance gains, in particular, in real-time applications or in highly compute-intensive tasks such as the building of probabilistic roadmaps [10].

Finally, the method is exact: for instance, a desired position can be reached exactly, and not only approached iteratively "as close as we want". This may have important consequences. For example, in the initial trajectory planning, one would no longer need to spend time finding a trajectory that ends very close to the target. Instead, one can plan a trajectory that ends roughly somewhere near the target, and then make an affine deformation towards the exact target position. In a similar vein, gap-reduction algorithms [3] may also substantially benefit from our method.

A last word on the biological implications of the ideas presented here. One source of inspiration for the present work was indeed the recent studies of affine invariance in human perception and movements (see e.g. [U] and references therein). Conversely, one could ask (and experimentally test) whether humans use algorithms similar to those described here to correct their hand or locomotor trajectories.

\section{Acknowledgments}

The author is deeply grateful to Prof D. Bennequin, Prof Y. Nakamura, and Dr O. Kanoun for their highly valuable suggestions and comments. This research was funded by an University of Tokyo grant and by a JSPS postdoctoral fellowship. 


\section{APPENDIX}

\section{A. Correcting the final orientation of the bicycle}

Remark that, if $\beta=0$ in equation (III), the final position $\mathcal{C}(T)$ does not move when $\lambda$ varies. However, the final orientation does vary with $\lambda$. Exploiting this fact, one can make corrections of the final orientation without changing the final position.

As remarked earlier, $\beta=0$ when $\overrightarrow{\mathcal{C}(\tau) \mathcal{C}(T)}$ and $\mathbf{v}(\tau)$ are collinear, that is, when the tangent line at time $\tau$ goes through $\mathcal{C}(T)$. Consequently, in order to make a correction of the final tangent vector from $\mathbf{u}_{\|}(T)$ to a desired tangent vector $\mathbf{u}_{d}$ while keeping the final position unchanged, it suffices to (see Fig. [, left plot)

1) find a time instant $\tau$ when the tangent line goes through $\mathcal{C}(T)$;

2) compute the appropriate $\lambda$ (see below);

3) make the affine deformation of parameter $\lambda$ at time $\tau$.
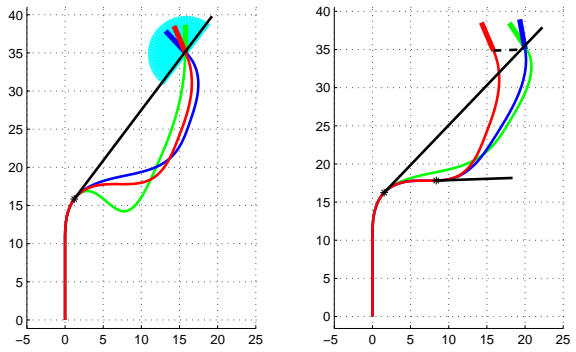

Fig. 5. Left: accessible final orientations (in cyan) and two examples of orientation corrections. The black line represents the tangent at $\tau$. Note that the black line goes through the final position, which remains unchanged by the orientation corrections. Right: correction of the final position and orientation.

Computation of $\lambda$ : Remark that the final orientation of the deformed trajectory is given by the vector $\mathcal{M}\left(\mathbf{u}_{\|}(T)\right)$. Observe next that

$$
\mathcal{M}\left(\mathbf{u}_{\|}(T)\right)=\mathbf{u}_{\|}(T)+\lambda \gamma \mathbf{v}(\tau)
$$

where $\gamma$ is the coefficient multiplying $\mathbf{a}(\tau)$ in the decomposition of $\mathbf{u}_{\|}(T)$ in the basis $\{\mathbf{v}(\tau), \mathbf{a}(\tau)\}$.

Consider $I$ the intersection between the line containing $\mathbf{u}_{d}$ and the line parallel to $\mathbf{v}$ and which goes through the tip of $\mathbf{u}_{\|}(T)$ (see illustration in Fig. 6 ). The directed distance between $I$ and the tip of $\mathbf{u}_{\|}(T)$ is given by

$$
d=\frac{\sin \left(\mathbf{v}\left(\widehat{\tau), \mathbf{u}_{\|}}(T)\right)\right.}{\tan \left(\widehat{\mathbf{v}(\tau), \mathbf{u}_{d}}\right)}-\cos \left(\mathbf{v}\left(\widehat{\tau), \mathbf{u}_{\|}}(T)\right) .\right.
$$

The appropriate $\lambda$ must then satisfy $\lambda \gamma \overline{\mathbf{v}(\tau)}=d$, which leads to $\lambda=d /(\gamma \overline{\mathbf{v}(\tau)})$. $\triangle$

Accessible orientations: The accessible orientations are restricted to the half-circle defined by the tangent line and in which lies $\theta(T)$, as shown in Fig. [1. Different choices of the tangent lines (when there exist more than one possible tangent line) induce different sets of accessible orientations, whose union forms the total set of accessible orientations.

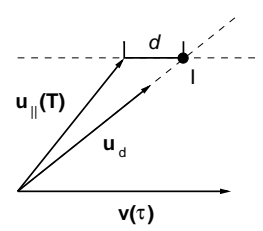

Fig. 6. Illustration for the computation of $\lambda$ in the correction of the final orientation.

Note that composing several deformations allows bypassing these restrictions. $\triangle$

Correcting the final position and orientation: Combining the previous development and the results of section $\mathbb{D - A} 3$ allows making corrections towards (almost) any positions and orientations in space as follows

1) correct the final position towards the desired position;

2) from the deformed trajectory, correct the final orientation towards the desired orientation while keeping the final position unchanged.

Fig. 1, right plot, shows an example of correction of both the final position and orientation. $\triangle$

\section{REFERENCES}

[1] D. Bennequin, R. Fuchs, A. Berthoz, and T. Flash. Movement timing and invariance arise from several geometries. PLoS Comput Biol, 5(7):e1000426, Jul 2009. doi: 10.1371/journal. pcbi. 1000426 .

[2] J.D. Boissonnat, A. Cerezo, and J. Leblond. A note on shortest paths in the plane subject to a constraint on the derivative of the curvature. Rapports de recherche-INRIA, 1994. ISSN 02496399.

[3] P. Cheng, E. Frazzoli, and S. LaValle. Improving the performance of sampling-based motion planning with symmetrybased gap reduction. IEEE Transactions on Robotics, 24(2): 488-494, 2008. ISSN 1552-3098.

[4] A. Filippov. Differential equations with discontinuous righthand sides. Springer, 1988. ISBN 902772699X.

[5] T. Fraichard and A. Scheuer. From Reeds and Shepp's to continuous-curvature paths. IEEE Transactions on Robotics, 20(6):1025-1035, 2004. doi: 10.1109/TRO.2004.833789.

[6] A. Kelly and B. Nagy. Reactive nonholonomic trajectory generation via parametric optimal control. The International Journal of Robotics Research, 22(7-8):583, 2003. ISSN 02783649.

[7] M. Khatib, H. Jaouni, R. Chatila, and J.P. Laumond. Dynamic path modification for car-like nonholonomic mobile robots. In IEEE International Conference on Robotics and Automation, 1997. ISBN 0780336127.

[8] F. Lamiraux, D. Bonnafous, and O. Lefebvre. Reactive path deformation for nonholonomic mobile robots. IEEE Transactions on Robotics, 20(6):967-977, 2004. doi: .829459.

[9] J.-P. Laumond. Robot Motion Planning and Control. SpringerVerlag, New York, 1998.

[10] S.M. LaValle and J.J. Kuffner. Randomized kinodynamic planning. The International Journal of Robotics Research, 20 (5):378, 2001. ISSN 0278-3649.

[11] Y. Nakamura and S. Savant. Nonlinear tracking control of autonomous underwater vehicles. In IEEE International Conference on Robotics and Automation, 1992. doi: 10.1109/ROBOT. 1992.220005.

[12] K. Seiler, S. Singh, and H. Durrant-Whyte. Using Lie group symmetries for fast corrective motion planning. In Algorithmic Foundations of Robotics IX, 2010. 